\title{
Actualidad de los cementos reparadores endodónticos: MTA y biodentine
}

\author{
Topicality of Endodontic Repairing Cements: MTA vs biodentine
}

Ulises Massino Peñaloza De La Torre ${ }^{1 a}$, Naysha Calizaya Laquise ${ }^{1 b}$

(D) https://orcid.org/0000-0002-8510-2350 (D) https://orcid.org/0000-0002-0564-8215

\section{Resumen}

Este artículo de revisión bibliográfica tuvo por objetivo presentar el material con mejores propiedades clínicas para realizar recubrimiento pulpar directo. En la actualidad, es de mucho interés e importancia mantener la vitalidad pulpar de los dientes; consecuentemente, se necesitan materiales odontológicos adecuados que posean propiedades que estimulen o que por lo menos no interfieran en la formación del puente dentinario en las lesiones pulpares. Por un lado, EI MTA es un material que ha ido mejorando sus propiedades físico - mecánicas. Por otro lado, el biodentine es un material relativamente nuevo usado como material de recubrimiento pulpar que posee propiedades similares al MTA, pero que tiene la ventaja de poseer un tiempo de fraguado más corto y mejor resistencia mecánica.

Palabras Clave: recubrimiento de la pulpa dental, remineralización dental (DeCS), pulpa dental

\section{Abstract}

The objective of this bibliographic review article was to present the material with the best clinical properties for direct pulp capping. At present, it is of great interest and importance to maintain the pulp vitality of the teeth; consequently, suitable dental materials are needed that possess properties that stimulate or at least do not interfere with the formation of the dentin bridge in pulp lesions. On the one hand, MTA is a material that has been improving its physical-mechanical properties. On the other hand, biodentine is a relatively new material used as a pulp capping material that has similar properties to MTA, but it has the advantage of having a shorter setting time and better mechanical resistance.

Keywords: dental pulp coating, dental remineralization (DeCS), dental pulp

\section{Introducción}

Las terapias de recubrimiento pulpar directo utilizan biomateriales para preservar y proteger los tejidos pulpares expuestos. De esta forma, se trata de inducir la reparación mediante la producción de una barrera mineralizada en la pulpa. ${ }^{1}$ El primer medicamento utilizado para el tratamiento de las heridas pulpares fue el hidróxido de calcio, introducido por B. W. Hermann, en el año $1920{ }^{2}$ Este polvo posee propiedades importantes como un $\mathrm{pH}$ alcalino de 12,4 que le confiere propiedades bactericidas. El hidróxido de calcio, al inicio de la terapia pulpar, produce inflamación leve, lo cual provoca una necrosis superficial y la formación del puente dentinario cuando es colocado directamente sobre la pulpa. $^{3} \mathrm{El}$

\footnotetext{
${ }^{1}$ Escuela Profesional de Odontología, Universidad Nacional Jorge Basadre Grohmann, Tacna, Perú

${ }^{a}$ Docente, Especialista en Periodoncia e Implantología

${ }^{\mathrm{b}}$ Estudiante
} 
tejido pulpar juega un papel importante en la producción de dentina secundaria y terciaria o de reparación. ${ }^{4}$

El agregado de trioxido mineral (MTA) fue el primer material biocerámico de este tipo patentado por Torabinejad en 1995. EI MTA presenta propiedades de biocompatibilidad y bioactividad, lo cual posibilita su uso para realizar recubrimientos pulpares directos. ${ }^{5}$ Además, se ha demostrado que estimula la formación de dentina debido a su alcalinidad, biocompatibilidad y propiedades remineralizantes. ${ }^{3}$

En el 2011 se desarrolló el biodentine, un silicato de calcio que puede ser usado como sustituto de dentina. Debido a sus propiedades mecánicas mejoradas y a su rápido fraguado, en comparación a otros, este silicato puede ser utilizado como un material definitivo de sustitución de dentina tanto coronal como radicular. ${ }^{5}$ Por tanto, el objetivo de la presente revisión bibliográfica es mostrar el material con mejores propiedades clínicas para realizar un recubrimiento pulpar directo.

\section{Método}

Se realizó una búsqueda bibliográfica durante el periodo comprendido entre el 4 de octubre de 2019 al 15 de mayo de 2020. En una primera búsqueda que incluía artículos de revisión y reporte de caso, se encontraron 200 artículos; seguidamente, se procedió a discriminar aquellos artículos que no permitian responder a la siguiente interrogante: ¿cuál es el material con mejores propiedades clínicas para realizar un recubrimiento pulpar directo?

La información de la presente revisión bibliográfica se obtuvo a partir de 24 artículos de la base de datos Pubmed, Google Académico y Scielo. La búsqueda se realizó ingresando las siguientes palabras clave: mineral trioxide aggregate, biodentine, tricalcium silicate, calcium hydroxide, dental pulp capping, dental pulp, tooth remineralization, calcium silicate cements.

Este artículo se realizó para conocer qué material tiene las mejores propiedades físicas para usarlo en las prácticas clínicas de los odontólogos como recubrimiento pulpar.
Mente et al. ${ }^{6}$ evaluaron una cantidad de pacientes en los que se hicieron protección pulpar directa tras una exposición pulpar. Los investigadores usaron dos materiales: hidróxido de calcio y MTA; y reportaron que el MTA parece ser más efectivo para un recubrimiento pulpar directo. Similares resultados obtuvieron Hilton et al., ${ }^{7}$ en un estudio clínico randomizado. Los autores reportaron que existe una menor probabilidad de fracaso en dientes tratados con MTA (19,7\%), al compararlo con hidróxido de calcio $(31,5 \%)$. En este estudio se incluyó una muestra extensa de 376 pacientes, quienes fueron controlados hasta por 2 años.

En una revisión de literatura publicada en el 2019 por Llanos, ${ }^{5}$ se demuestra que las propiedades de resistencia a la compresión está relacionada a la etapa de hidratación y presión durante la aplicación. Mientras que la solubilidad puede asociarse al óxido de bismuto. (concetor) El potencial de hidrogeniones $(\mathrm{pH})$ puede elevarse a 12,5 a las 3 horas después de la mezcla.

Anabela Paula et al. ${ }^{8}$ evaluaron la citotoxicidad y la bioactividad de tres materiales diferentes de recubrimiento pulpar directo, tales como hidróxido de calcio, agregado de trióxido mineral y silicato de calcio en una línea celular de ratón tipo odontoblastos. Los autores concluyeron que los resultados de MTA confirmaron su indicación para estas terapias, y justificaron su reconocimiento como el "estándar de oro". El biodentine puede ser una alternativa, ya que promueven la misma respuesta celular que el agregado de trióxido mineral (MTA).

En un estudio realizado por Tran et al. ${ }^{9}$ se estudió la composición química y la microestructura del puente dentinario después del recubrimiento pulpar directo con biodentine y MTA en 14 ratas. Luego de 30 días, se realizó una evaluación histológica mediante microscopia electrónica y microanálisis de Rx. Los investigadores reportaron que los cementos a base de silicato de calcio utilizados como materiales de recubrimiento pulpar proporcionaron un entorno óptimo para la cicatrización pulpar, lo que resultó en una dentina reparadora que se asemeja en ciertos puntos a la dentina. 


\section{Agregado de trioxido mineral}

Es un cemento a base de silicato de calcio. ${ }^{10}$ Es un material hidrofílico ideal para procedimientos clínicos como un recubrimiento pulpar directo. ${ }^{11}$ El desarrollo de este material, ha sido probado en el laboratorio, en estudios con animales y luego en ensayos clínicos. Los resultados clínicos satisfactorios, debido a la cualidad de este material en estimular la reparación de la herida pulpar, hicieron que el MTA se convierta en un material de uso común. ${ }^{12,13}$ Este silicato fue el primero desarrollado y patentado por Torabinejad y White en $1995 .{ }^{5}$ "Gray MTA" (GMTA) fue el primero en introducirse, y el MTA blanco (WMTA) se desarrolló algunos años después. Esta última versión hizo que se mejorara la estética en el material. ${ }^{14}$ Los estudios que comprar aron su composición concluyeron que la diferencia de color entre estas dos versiones se debe a la falta de compuestos de hierro en la fórmula.

\section{Composición}

Su principal componente es cemento de silicato de calcio, al cual se le ha agregado óxido de bismuto para efectos de radiopacidad. Se ha encontrado que la radiopacidad media para MTA es de $7,17 \mathrm{~mm}$ de espesor equivalente de aluminio. EI MTA inicialmente era una mezcla mecánica de tres ingredientes en polvo: cemento portland (75\%), óxido de bismuto $(20 \%)$ y yeso $(5 \%) .{ }^{12}$ Actualmente, los principales componentes del GMTA son: silicato tricalcico, silicato dicálcico y oxido de bismuto. Mientras que los componentes de la versión blanca, WMTA, son silicato tricálcico y óxido de bismuto. ${ }^{26}$

\section{Propiedades}

Las principales propiedades que tiene el MTA son su biocompatibilidad, favorecida por su citotoxicidad baja; así como la inducción de la proliferación y adhesión celular y expresión adecuada de citocinas inflamatorias durante su contacto con la pulpa e inflamación pulpar reducida luego de la fase aguda. ${ }^{15}$

\section{Manipulación}

Dado la consistencia granular del MTA, se hace un poco difícil su manipulación y aplicación en el sitio clínico. ${ }^{16}$

\section{Biodentine}

Biodentine ha sido reconocido en la literatura como un biomaterial representante de los cementos a base de silicato tricálcico, actualmente utilizados en odontología. ${ }^{17}$ Este material tiene un potencial antiinflamatorio y a la vez tiene la propiedad de inducir la capacidad de regeneración de la pulpa. ${ }^{18}$

El biodentine presenta un menor tiempo de fraguado, comparado con los silicatos de calcio tradicionales debido al tamaño más pequeño de las partículas del polvo. Esta condición permitiría una mayor área de reacción por la adición de la solución acuosa de cloruro de calcio que es un potente acelerador de la reacción en estos materiales. ${ }^{5}$ Con esto, el biodentine obtuvo una mejora en comparación con el MTA respecto al tiempo de fraguado. ${ }^{19}$

\section{Presentación y modo de empleo}

La presentación de biodentine es en forma de una cápsula que contiene el polvo y un líquido contenido en una ampolla. Dependiendo de la preferencia, puede aplicarse con un porta amalgama o una espátula. ${ }^{5}$

\section{Composición}

La composición del polvo es el silicato tricálcico $(80,1 \%)$, carbonato de calcio $(14,9 \%)$, óxido de circonio (5\%) y óxido de hierro, mientras que el líquido contiene cloruro de calcio que actúa como un acelerador. ${ }^{19}$

\section{Propiedades}

Su mejor manipulación, es una de sus principales propiedades; además del tiempo de fraguado más rápido, mejor resistencia a la compresión, porosidad disminuida y síntesis temprana de dentina reparativa, en comparación al MTA. Asimismo, la acción antibacteriana de este biomaterial está determinada por los componentes de calcio, los cuales se convierten en soluciones acuosas de hidróxido de calcio. ${ }^{20}$ Por su bioactividad, el biodentine se puede considerar como un material apropiado para la regeneración del complejo dentinopulpar en recubrimientos pulpares directos, pues no induce daño a las células pulpares; además, estimula la formación de dentina reparadora. ${ }^{21}$ 
Awawdeh ${ }^{23}$ estudió las tasas de éxito del biodentine y MTA usados como recubrimiento pulpar directo en 3 años de seguimiento. El estudio concluyó que el biodentine y MTA tienen tasas de éxito favorables, coincidiendo con la revisión sistemática con metaanálisis realizada por Paula et al. ${ }^{1}$ que comparó la efectividad de los biomateriales usado como recubrimiento pulpar directo, no encontrando diferencias estadísticamente significativas en la tasa de éxito.

En un ensayo clínico aleatorizado realizado por Parinyaprom et al. ${ }^{22}$ donde también evaluaron la decoloración gris provocada por biodentine y MTA. Cuando se usó biodentine como material de recubrimiento pulpar directo encontraron que este biomaterial no causó ninguna decoloración gris; por lo que puede ser usado en dientes permanentes en zona estética, en exposiciones de hasta $2,5 \mathrm{~mm}$.

Nowicka et al. ${ }^{24}$ realizaron evaluaciones tomográficas del volumen $\mathrm{y}$ densidad del puente dentinario luego de 6 semanas del recubrimiento pulpar directo con MTA, biodentine y Single Bond Universal en dientes humanos. Los autores concluyeron que el biodentine y MTA indujeron la formación de puentes con un volumen promedio significativamente mayor en comparación con Single Bond Universal.

En la revisión actualizada de literatura de los últimos tres años por Rajasekharany et al., ${ }^{17}$ se indica que las propiedades físicas y biológicas mejoradas de biodentine tiene un gran potencial para revolucionar las diferentes modalidades de tratamiento en odontopediatría y endodoncia, especialmente después de lesiones traumáticas.

Los autores Paula et al. ${ }^{8}$ evaluaron la citotoxicidad y la bioactividad de hidróxido de calcio, MTA y biodentine como materiales de recubrimiento pulpar directo. La investigación concluyó que el uso del MTA está justificando y su reconocimiento como el "estándar de oro"; el biodentine puede ser una alternativa, ya que promueven la misma respuesta celular que el agregado de trióxido mineral.

\section{Conclusión}

Los diversos estudios encontrados sugieren que ambos materiales, MTA y biodentine, son recomendables como recubrimiento pulpar directo dado que poseen tasas de éxito similares. Por ejemplo, no producen coloración gris en dientes anteriores debido a sus propiedades mejoradas, producen un puente dentinario de configuración adecuado; además, producen una citotoxicidad similar, manteniendo la vitalidad pulpar. Sin embargo, el biodentine presenta un tiempo menor de fraguado y mejores propiedades mecánicas.

\section{Referencias}

1. Paula $A B$, Laranjo $M$, Marto $C M$, Paulo S, Abrantes AM, Casalta-Lopes J, Marques-Ferreira M, Botelho MF, Carrilho E. Direct Pulp Capping: What is the Most Effective Therapy?Systematic Review and Meta-Analysis. J Evid Based Dent Pract. Dic 2018;1 $8(4): 298-314$. D o i : 10.1016/j.jebdp.2018.02.002.

2. Bravo A, Diaz D, Yupanqui K, Mendiola C. Apicogénesis en canino permanente joven con resorción intracoronal pre eruptiva: reporte de caso. Rev Estomatol Herediana. Ene-Mar 2019; $29(1): 80-88$. D o i : 10.20453/reh.v29i1.3497

3. Yepes Delgado FL, Castrillón Yepes CA. Calcium hydroxide as a clinical paradigm is surpassed by mineral trioxide aggregate (MTA). Rev Fac Odontol Univ Antioquia. diciembre de 2013; 25(1):176-207. ISSN 0121-246X

4. Adıgüzel $M$, Ahmetoğlu $F$, Eldeniz AÜ, Tekin MG, Göğebakan B. Comparison of cytotoxic effects of calcium silicatebased materials on human pulp fibroblasts Mehmet. J Dent Res Dent Clin Dent Prospects. 2019; 13(4):241246. Doi: 10.15171/joddd.2019.037.

5. Llanos M, Evolución de los cementos biocerámicos en endodoncia. Conocimiento para el Desarrollo, 2019, [Internet]. febrero de 2016 [citado 1 de noviembre de 2019]; , 10(1): 151- 
162.DOI: 10.17268/CpD.2019.01.24

6. Mente J, Geletneky B, Ohle M, Koch MJ, Friedrich Ding PG, Wolff D, Dreyhaupt J, Martin N, Staehle HJ, Pfefferle T. Mineral trioxide aggregate or calcium hydroxide direct pulp capping: an analysis of the clinical treatment outcome. J Endod. May $2010 ; 36(5): 806-13$. Doi: 10.1016/j.joen.2010.02.024.

7. Hilton TJ, Ferracane JL, Mancl L; Northwest Practice-based Research Collaborative in Evidence-based Dentistry (NWP). Comparison of $\mathrm{CaOH}$ with MTA for direct pulp capping: a PBRN randomized clinical trial. J Dent Res. Jul 2013; 92(7 Suppl):16S-22S. Doi: $10.1177 / 0022034513484336$.

8. Paula A, Laranjo M, Marto CM, Abrantes AM, Casalta-Lopes J, Gonçalves AC, Sarmento-Ribeiro AB, Ferreira MM, Botelho MF, Carrilho E. B i o d e $n t$ i n e TM B o o s $t s$, WhiteProRoot@MTA Increases and Life $®$ Suppresses Odontoblast Activity. Materials (Basel). 11 Apr 2019; $12(7): 1184$. D o i : 10.3390/ma12071184.

Tran XV, Salehi H, Truong MT, Sandra M, Sadoine J, Jacquot B, et al. Reparative Mineralized Tissue Characterization after Direct Pulp Capping with CalciumSilicate-Based Cements. Mater Basel Switz. 29 de junio de 2019; 12(13): 2102. Doi: $10.3390 / \mathrm{ma1} 12132102$.

9. Dawood AE, Parashos P, Wong RHK, Reynolds EC, Manton DJ. Calcium silicate-based cements: composition, properties, and clinical applications. J Investig Clin Dent. May 2017;8(2). Doi: 10.1111/jicd.12195.

10. Guimarães BM, Vivan RR, Piazza B, Alcalde MP, Bramante CM, Duarte $\mathrm{MAH}$. Chemical-physical properties and apatite-forming ability of mineral trioxide aggregate flow. $\mathrm{J}$ Endod. Oct $2017 ; 43$ (10): $1692-6$. Doi : 10.1016/j.joen.2017.05.005.

11. Ha WN, Nicholson T, Kahler B, Walsh LJ. Mineral Trioxide Aggregate-A
Review of Properties and Testing Methodologies. Mater Basel Switz. 2 nov 2017;10(11):1261. Doi: 10.3390/ma10111261.

12. Ghilotti J, Sanz JL, López-García S, Guerrero-Gironés J, Pecci-Lloret MP, Lozano A, Llena C, Rodríguez-Lozano FJ, Forner L, Spagnuolo G. Comparative Surface Morphology, Chemical Composition, and Cytocompatibility of Bio-C Repair, Biodentine, and ProRoot MTA on hDPCs. Materials (Basel). 10 May $2020 ; 13(9): 2189$. Do i : $10.3390 / m a 13092189$.

13. Mahmoud SH, El-Negoly SA, Zaen ElDin AM, El-Zekrid MH, Grawish LM, Grawish HM, Grawish ME. Biodentine versus mineral trioxide aggregate as a direct pulp capping material for human mature permanent teeth - A systematic review. J Conserv Dent. Sept-oct $2018 ; 21(5): 466-473$. Doi: 10.4103/JCD.JCD_198_18.

14. de Oliveira NG, de Souza Araújo PR, da Silveira MT, Sobral APV, Carvalho M de V. Comparison of the biocompatibility of calcium silicate-based materials to mineral trioxide aggregate: Systematic review. Eur J Dent. junio de $2018 ; 12$ ( 2$): 317-26$. D o i : 10.4103/ejd.ejd_347_17.

15. Zafar K, Jamal S, Ghafoor R.Bio-active cements-Mineral Trioxide Aggregate based calcium silicate materials: a narrative review. J Pak Med Assoc. Mar $2020 ; 70(3): 497-504$. Doi: 10.5455/JPMA.16942. PMID: 32207434.

16. Rajasekharan S, Martens LC, Cauwels RGEC, Anthonappa RP. Biodentine ${ }^{\mathrm{TM}}$ material characteristics and clinical applications: a 3 year literature review and update. Eur Arch Paediatr Dent. Feb 2018; 19(1):1-22. Doi: 10.1007/s40368-018-0328-x. Epub 25 Jan 2018. Erratum in: Eur Arch Paediatr Dent. 15 Mar 2018;: Erratum in: Eur Arch Paediatr Dent. 10 sept 2020.

17. Giraud T, Jeanneau C, Rombouts C, Bakhtiar H, Laurent P, About I. Pulp 
capping materials modulate the balance between inflammation and regeneration. Dent Mater Off Publ Acad Dent Mater. 2019; 35(1):24-35. Doi: 10.1016/j.dental.2018.09.008

18. Kaur M, Singh $H$, Dhillon JS, Batra M, Saini M. MTA versus Biodentine: Review of Literature with a Comparative Analysis. J Clin Diagn Res JCDR. Agt 2017;11(8):ZG01-5. D O i 10.7860/JCDR/2017/25840.10374.

19. Narváez SH, Rodríguez ALV. Biodentine: Un nuevo material en terapia pulpar / Biodentine: A New Material for Pulp Therapy. Niv Odontol. Jul-dic 2015; 34(73): 69-76. ISSN 0120-4319

20. Laurent $\mathrm{P}$, Camps J, De Méo M, Déjou $\mathrm{J}$, About I. Induction of specific cell responses to a $\mathrm{Ca}(3) \mathrm{SiO}(5)$-based posterior restorative material. Dent Mater Off Publ Acad Dent Mater. Nov 2008 ; 24(11):1486-94. Doi: 10.1016/j.dental.2008.02.020.

21. Parinyaprom N, Nirunsittirat A, Chuveera P, Na Lampang S, Srisuwan T, Sastraruji T, Bua-On P, Simprasert S, Khoipanich I, Sutharaphan T, Theppimarn S, Ue-Srichai N, Tangtrakooljaroen W, Chompu-Inwai P. Outcomes of Direct Pulp Capping by Using Either ProRoot Mineral Trioxide Aggregate or Biodentine in Permanent Teeth with Carious Pulp Exposure in 6to 18-Year-Old Patients: A Randomized Controlled Trial. J Endod. Mar 2018;
44 ( 3 ): $341-348$. D o i : 10.1016/j.joen.2017.10.012

22. Awawdeh L, Al-Qudah A, Hamouri H, Chakra RJ. Outcomes of Vital Pulp Therapy Using Mineral Trioxide Aggregate or Biodentine: A Prospective Randomized Clinical Trial. J Endod. Nov 2018; 44(11):1603-9. Doi: 10.1016/j.joen.2018.08.004.

23. Nowicka A, Wilk G, Lipski M, Kołecki J, B u c zkowska-Radlińska J. Tomographic Evaluation of Reparative Dentin Formation after Direct Pulp Capping with $\mathrm{Ca}(\mathrm{OH}) 2, \mathrm{MTA}$, Biodentine, and Dentin Bonding System in Human Teeth. J Endod. Agto $2015 ; 41(8): 1234-40$. Doi : 10.1016/j.joen.2015.03.017

24. Mahmoud SH, El-Negoly SA, Zaen ElDin AM, El-Zekrid MH, Grawish LM, Grawish HM, Grawish ME. Biodentine versus mineral trioxide aggregate as a direct pulp capping material for human mature permanent teeth - A systematic review. J Conserv Dent. Sept-oct 2018; 21(5):466-473. Doi: 10.4103/JCD.JCD_198_18.

25. Corral-Núñez $\mathrm{C}$, Fernández-Godoy $\mathrm{E}$, Martin-Casielles J, Estay J, BersezioMiranda C, Cisternas-Pinto $P$ et al Revisión del estado actual de cementos de silicato de calcio en odontología restauradora. Rev Fac Odontol Univ Antioq 2016; 27(2): $425-441$. D o i : 10.17533/udea.rfo.v27n2a10
Correspondencia: ulisespe@hotmail.com
Fecha de recepción : 16 de junio de 2020

Fecha de aceptación : 02 de septiembre de 2020 